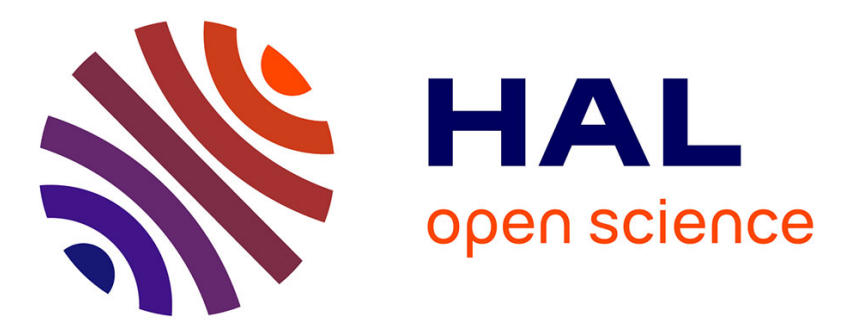

\title{
La mémoire et le corps dans l'expérience des mystiques
} Jean-Pierre Albert

\section{To cite this version:}

Jean-Pierre Albert. La mémoire et le corps dans l'expérience des mystiques. La mémoire de récit, May 2000, Bordeaux, France. halshs-00369923

\section{HAL Id: halshs-00369923 https://shs.hal.science/halshs-00369923}

Submitted on 23 Mar 2009

HAL is a multi-disciplinary open access archive for the deposit and dissemination of scientific research documents, whether they are published or not. The documents may come from teaching and research institutions in France or abroad, or from public or private research centers.
L'archive ouverte pluridisciplinaire HAL, est destinée au dépôt et à la diffusion de documents scientifiques de niveau recherche, publiés ou non, émanant des établissements d'enseignement et de recherche français ou étrangers, des laboratoires publics ou privés. 


\section{La mémoire et le corps dans l'expérience des mystiques}

Colette de Corbie, la réformatrice de l'ordre des Clarisses, au XVe siècle, revivait dans sa chair les supplices des martyrs au cours de la nuit qui précédait leur fête. « Elle se sentait tantôt brûlée, comme les bienheureux diacres Laurent et Vincent, tantôt écorchée comme saint Barthélemy, tantôt crucifiée, coupée en morceaux, plongée dans l'eau ou l'huile bouillante. Parfois il lui semblait qu'on lui arrachait le cœur, qu'on lui ouvrait et déchirait les entrailles, qu'on enfonçait dans ses chairs des tiges enflammées, et qu'on la transperçait de part en part (Ribet, 1895, II : 495)». La même chose arrivait à une obscure mystique bretonne du XVIIe siècle, Marie-Amice Picard, au point qu'on put la désigner comme « un martyrologe vivant (Le Gouvello, $1913: 251) »^{1}$.

Marie d'Agréda (1970), Anne-Catherine Emmerich et quelques autres, pour leur part, eurent des visions ou révélations de la vie du Christ et de la Vierge qui, une fois transcrites, représentent des centaines de pages de texte. La seconde fut par ailleurs une des stigmatisées les plus célèbres du début du XIXe siècle (Schmoeger, 1868-72).

Je n'ai cité ici que des cas extrêmes de phénomènes très communs dans l'univers de la mystique : un nombre considérable de saintes ont revécu les souffrances de la Passion ou recueilli à leur propos des révélations inédites au cours de leurs extases. Le point commun à ces deux types de données est qu'il s'agit toujours d'actualisations d'un récit fondateur : œuvres de la mémoire, si l'on veut, mais d'une mémoire qui n'est pas reconnue comme telle, ou qui soumet le récit appris à des restitutions bien singulières. Cela invite à s'interroger sur les modalités de la «mémoire du récit» dans le contexte d'une religion qui, fondée sur des récits, a conçu le cœur de sa liturgie comme une commémoration - «Faites ceci en mémoire de moi ». On va le voir, la simplicité apparente de ce schéma invite à s'interroger sur le statut même du récit et de la mémoire dans le cadre du christianisme, ou plus précisément du catholicisme.

\section{Des récits « agissants »}

\footnotetext{
${ }^{1}$ Pour une analyse plus développée de ces exemples, voir ALBERT, 1997.
} 
La lecture du texte biblique est soumise de longue date, dans les Eglises antérieures à la Réforme, à des contraintes herméneutiques dont la formulation la plus synthétique est la théorie des quatre sens de l'Ecriture (De Lubac, 1959) :

\section{Littera gesta docet, quid credas allegoria}

\section{Moralis quid agas, quo tendas anagogia}

Hors du sens littéral, qui seul est supposé instruire des res gestae, un récit biblique ne se livre donc pas à une simple attente d'information « historique », il devient le prétexte d'une méditation destinée à changer le lecteur : une bonne lecture doit susciter la foi, aider à la conduite de l'action et donner les clés du salut. Quel sens y aurait-il à ne retenir du texte que sa matière narrative, à se contenter de l'apprendre pour le dire à son tour ? C'est à l'existence du chrétien tout entière de témoigner de sa mémoire, la lettre du récit dût-elle être oubliée.

Plus généralement, un récit sacré - au premier chef celui de la Passion - est destiné à faire son chemin dans la conscience et dans la vie de son destinataire. En connaître la lettre n'est que la première étape du déploiement inachevable de son sens et, surtout, du processus d'appropriation qui donnera à sa « compréhension » sa pleine densité existentielle. Cela prend souvent la forme d'une découverte soudain bouleversante de ce que l'on savait déjà, d'une « conversion », pour emprunter le langage des théologiens et des hagiographes. Dans les faits, le récit n'accomplit sa fonction que lorsqu'il convertit : le marchand lyonnais Valdes trouva ainsi sa vocation de réformateur religieux en entendant la Vie de saint Alexis, le bourgeois siennois Jean Colombini, fondateur de l'ordre éphémère des jésuates, en découvrant celle de sainte Marie Egyptienne (De Rambuteau, 1893). Dans les deux cas, la conversion est imitation: comme le noble Alexis et la riche courtisane, ces bourgeois opulents abandonneront tout pour suivre le Christ. Le récit leur a fourni un projet d'existence. De tels phénomènes ne sont pas réservés aux temps lointains du Moyen Age : l'autobiographie de sainte Thérèse de Lisieux a suscité la vocation d'un nombre considérable de religieuses au XXe siècle.

Agissant, le récit l'est encore dans la liturgie. Si l'on suit la théologie de l'eucharistie fixée par le Concile de Latran IV, les mots de la consécration ne commémorent pas la dernière scène et la Passion, ils la réactualisent. Une pédagogie du miracle eucharistique vient confirmer qu'il en est bien ainsi : le pain sacrificiel saigne ou devient chair. Mais c'est du côté de la vie des saints que l'on trouve les actualisations les plus littérales du sacrifice fondateur : leur souffrance n'est pas seulement une image de celle du Christ, elle s'ajoute à elle dans la réitération d'un sacrifice dont la théologie, à partir du XVIIe siècle surtout, explicitera les 
mérites. Alors le récit devient vie, et en retour la biographie mémoire : tel est le sens le plus haut de l'Imitatio Christi.

\section{La biographie et sa mise en forme}

Il faut encore se demander à quelles conditions un récit peut à ce point devenir agissant. Sa réception par les mystiques est assurément un cas limite, mais il est significatif de la manière dont le christianisme (et peut-être aussi toute autre religion) parvient à rendre présents ses mythes fondateurs.

La « conversion » des saintes mystiques, telle qu'on l'a évoquée, n'est que très rarement un coup de tonnerre inopiné dans le ciel serein d'une vie. La rupture qu'elle induit est presque toujours consécutive à des accidents biographiques douloureux: deuil, grave maladie, défection d'un fiancé... Il faut des vies brisées pour suivre Jésus, l'imiter jusqu'au bout et assumer un destin de victime. Le corps, le plus souvent, est là pour exprimer ce cheminement. Par la douleur, il favorise l'évocation de la Passion avant d'en devenir le mémorial.

« Dieu n'habite pas les corps bien portants », écrivait Hildegarde de Bingen au XIIe siècle. Et, dans les faits, les corps sur lesquels on veut lire les stigmates de la crucifixion sont presque toujours des corps défaits, ruinés par la maladie ou les exercices ascétiques. Le récit de la Passion vient ainsi donner forme à des existences tragiques qui trouvent en lui sens et justification. La «théologie de la réparation» et la «spiritualité victimale », largement diffusées depuis le XVIIe siècle, assurent aux vocations de victime une reconnaissance par leur entourage qui conforte l'interprétation qu'elles donnent elles-mêmes de leur situation : l'impuissance devient force, la dépendance action de portée infinie. La misérable grabataire est le Christ, elle œuvre secrètement au salut de tous.

Les stigmates offrent l'expression la plus figurative de ce rôle rédempteur. Soumis au cycle des commémorations liturgiques, ils saignent en général le vendredi, ou seulement au temps de la Semaine Sainte. Leur manifestation s'accompagne parfois d'une mise en scène quasi théâtrale. C'est ainsi que Marie de Moerl, une des très controversées « stigmatisées du Tyrol » en vogue au milieu du XIXe siècle, mimait avec emphase les temps forts de la vie du Christ devant les fidèles ébahis qui se pressaient dans sa chambre. A Noël elle berçait le nouveau-né, et le clou du spectacle était bien évidemment la Semaine Sainte, pendant laquelle elle mimait jour après jour les épisodes de la Passion (Boré, 1846). L'actualisation du récit culmine ainsi dans une sorte de théâtre de la possession, qui tend à effacer la distance entre le 
passé et le présent : à l'évocation mimétique de l'événement de référence se superpose, dans l'esprit des spectateurs, un nouvel événement, la «passion» actuelle d'une victime sacrificielle. Mais cette rencontre n'est possible que parce que, au Golgotha comme dans la ville autrichienne de Kaltern, l'événement lui-même est aussi une occurrence, indéfiniment réitérable, d'un unique scénario : celui, liturgique, cosmique et métaphysique, du sacrifice.

\section{Le récit et le rite}

La lecture sacrificielle de la vie et de la mort du Christ soulève une difficulté déjà sensible dans les textes évangéliques : du Christ ou de Judas, les acteurs principaux, peut-on jamais dire qu'ils veulent ce qu'ils font? ne se soumettent-ils pas plutôt à la nécessité (traduite dans certaines paroles de Jésus en prophéties) du scénario de la Rédemption ? Il est possible de concevoir la vie terrestre du Christ, ramenée dans les commentaires à l'unité d'un sens qui se manifeste dans la Passion, comme l'accomplissement d'une transformation métaphysique du monde dont l'équivalent le plus proche serait l'action rituelle, ou le sacrement. Et c'est là, me semble-t-il, la raison principale du recours au modèle du sacrifice, d'un rite donc, pour tenter de penser son efficacité.

Pousser cette logique jusqu'au bout invite à s'interroger sur la nature des liens qui unissent les événements pouvant faire l'objet d'un récit. Tout peut être raconté : l'évolution d'une maladie, un tirage du loto, une histoire d'amour, une partie de football, une bataille navale, le déroulement d'un procès, les funérailles d'un roi...D'un exemple à l'autre, les liens entre les séquences élémentaires des processus décrits varient considérablement : ils peuvent relever de la causalité naturelle, des lois de la psychologie, de l'application d'une règle de procédure, de l'obéissance à des ordres, d'une prescription rituelle, etc., ou de la conjonction de plusieurs de ces facteurs. On retiendra du moins que la succession des séquences d'un rituel se distingue fortement de la plupart des autres cas (à l'exception de la succession aléatoire) par son manque de motivation interne ${ }^{2}$. Au contraire, ce que l'on appelle d'ordinaire un récit correspond à un horizon d'attentes indexées sur l'ordre de la nature et de la société, ou sur une forme définie de liberté prise avec ces cadres (dans le cas, par exemple, de la littérature fantastique). L'étrangeté du mythe vient souvent de ce qu'il entrecroise ces registres, décrivant par exemple des phénomènes naturels sur le mode de l'autorité politique (cas de la création par la parole) ou de l'acte religieux (cas des cosmogonies sacrificielles). Plus généralement, le mythe soumet la succession des séquences narratives à des contraintes

\footnotetext{
${ }^{2}$ Voir à ce propos P. BOYER, 1997, ch. 7.
} 
sémantiques sans relation, pour certaines d'entre elles au moins, avec la conduite d'un récit bien construit. Et s'il est vrai, comme l'a suggéré Durkheim, qu'un mythe est souvent destiné à justifier un rituel, on comprend que l'arbitraire du second induise l'inconséquence du premier.

La projection de la logique du rituel dans une histoire supposée ou un récit des origines n'est donc pas une spécificité de la pensée chrétienne. Mais son génie est peut-être d'avoir emmêlé de façon inextricable ces types de processus. Le coup de force interprétatif qui fait reconnaitre un sacrifice dans un procès suivi d'une condamnation à mort induit une confusion entre rite et récit, scénario rituel et narration biographique, qui deviennent en définitive interchangeables. La chose est d'autant plus complexe que la notion même de sacrifice est inséparable d'une exégèse - au mieux un observateur non informé pourrait reconnaitre en présence de la mise à mort sacrificielle d'une victime une occurrence de «mise à mort cérémonielle en contexte religieux » qui pourrait aussi bien être un abattage rituel d'animaux à des fins alimentaires ou, s'il s'agit d'un homme, le châtiment d'un profanateur ou d'un régicide. A l'inverse, un rituel sémantiquement peu évocateur (comme la messe) peut être érigé par la glose indigène en rite sacrificiel.

On s'y perdrait à moins. Or la force des religions tient pour beaucoup à leur capacité de conduire la pensée à se perdre, assaillie de paradoxes sublimes et d'inépuisables non sens. Pour s'en tenir ici aux liens entre mémoire et récit, il est désormais clair que le récit de la Passion, dans la mesure où il est déjà celui d'une liturgie, admet sans difficulté des remémorations liturgiques très inégalement figuratives. Mais comme ce récit est en même temps biographique, il peut aussi être raconté et se prêter, comme n'importe quel récit de ce genre, aux projections ou identifications de ses lecteurs. Le cumul de ces deux dimensions liturgique et biographique - explique enfin la situation vécue par les mystiques : se sentir, dans un éternel présent, Christ et victime, tout en assurant, par la liturgie de leur propre sacrifice, le salut de l'humanité. En effet, le temps du rituel s'autonomise par rapport à celui de l'histoire, et c'est en ce sens que le Christ et ses imitatrices peuvent être indéfiniment recrucifiés. C'est en ce sens aussi que l'événement fondateur reste éternellement présent. Lorsqu'une mystique détaille ses visions de la crucifixion, c'est parce qu'elle y assiste ellemême. Même si, bien évidemment, elle ne fait que reproduire et amplifier un récit mille fois entendu, elle doit croire (et son entourage doit croire avec elle) qu'elle ne fait pas appel à sa mémoire, mais qu'elle est «inspirée ». Cette certitude est, du reste, encore plus grande lorsque la narratrice intarissable de la Passion est, elle-même, une souffrante à vocation 
sacrificielle. Le cas le plus exemplaire à cet égard est celui d'Anne-Catherine Emmerich, visionnaire et stigmatisée.

Ce qui précède invite donc à s'interroger sur le statut de la mémoire et de la commémoration dans le cadre du christianisme latin et du catholicisme. Il s'agit là sans aucun doute d'une question beaucoup plus large, celle des relations entre le mythe et le rite. Je m'en tiendrai ici à la manière dont elle se pose dans le cadre du (ou des) christianisme(s). La théologie de Calvin a simplifié le problème en récusant la lecture sacrificielle de l'eucharistie et en la réduisant à une pure commémoration. Quant au monde orthodoxe, il ignore le phénomène de la stigmatisation - peut-être parce qu'il dispose d'une théologie de l'image (de l'icône) qui reconnaît explicitement sa sacralité. Les faits cités ici posent donc un problème passionnant, dont je ne possède pas la solution : quelle est la singularité du catholicisme qui permet aux mystiques d'incarner les récits, de les revivre en un sens qui n'a rien de métaphorique ? Une partie de la réponse est sans doute à chercher dans la théologie - par exemple dans la définition du sacrement comme "signe efficace » réaffirmée au Concile de Trente, ou encore dans le rôle salvateur reconnu aux « œuvres". Mais il faut aussi se demander en quoi les cadres ainsi posés, actifs dans la mesure où ils commandent l'intervention de l'institution, rejoignent des formules religieuses beaucoup plus générales, par exemple les différentes modalités de la possession. Une chose semble du moins certaine : dès lors que le récit est celui d'un rite, et non d'une séquence historique, il se prête plus à l'action qu'à la récitation. On peut alors parler d'une « mémoire du récit » qui, conformément me semble-t-il à une construction sociologique (et non psychologique) de l'idée de mémoire, vaut comme matrice des comportements des individus ou des groupes, et non comme simple capital mémoriel appelant des performances narratives. Pour pasticher une formule célèbre, on pourrait dire que la mémoire, ainsi conçue, est ce qui reste quand on a tout oublié.

\section{Bibliographie}

ALBERT, Jean-Pierre, 1997, Le sang et le Ciel. Les saintes mystiques dans le monde chrétien, Paris, Aubier. 


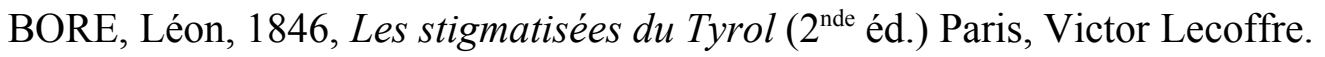

BOYER, Pascal, 1997, La religion comme phénomène naturel, Paris, Bayard Editions.

LE GOUVELLO, Hippolyte, Armelle Nicolas, dite la Bonne Armelle, Paris, Téqui.

LUBAC, Henri de, 1959, Exégèse médiévale. Les quatre sens de l'Ecriture, $1^{\text {ìre }}$ partie, Paris, Aubier, 2 vol.

Marie d'Agréda, 1970 (réed.), La cité mystique de Dieu, Saint-Céneré, Editions SaintMichel, 3 vol.

RAMBUTEAU, (Comtesse de), 1893, Le Bienheureux Colombini. Histoire d'un Toscan au XIVe siècle (3 $3^{\text {ème }}$ ed.), Paris, Victor Lecoffre.

RIBET, Joseph, 1895, La mystique divine distinguée des contrefaçons diaboliques et des analogies humaines, Paris, Poussielgue, 2 vol.

SCHMOEGER, Eugen, 1868-72, Vie de Anne-Catherine Emmerich, trad. M. de Cazales, Paris, Ambroise Bray, 3 vol. 